\title{
Valence-band and surface electronic structure of CdTe
}

\author{
K. O. Magnusson and S. A. Flodström* \\ MAX-lab, Lund University, P.O. Box 118, S-221 00 Lund, Sweden \\ P. E. S. Persson \\ Department of Physics and Measurement Technology, Linköping University, S-581 83 Linköping, Sweden
}

(Received 16 February 1988)

\begin{abstract}
Angle-resolved uv photoelectron spectroscopy has been used to study the electronic structure of cleaved CdTe. Within a direct-transition model, valence-band states on the $\Gamma-(K)-X$ line are probed in normal emission, yielding the energy of the critical points $\Gamma_{7}$ and $\Gamma_{8}$ and of the minimum of the spin-orbit-split second valence band. A relativistic augmented-plane-wave calculation including the effects of the $\mathrm{Cd} 4 d$ states has been performed and is favorably compared with the experimental results. Through this comparison, the final-state bands are found to be well approximated with free-electron bands in the normal direction and in a $\langle 111\rangle$ direction. Through extensive studies in off-normal emission at selected photon energies and under specific polarization geometries, two surface resonances located (in energy) close to, but below the valence-band edge and two surface states found in the open lens of the calculated projected valence-band structure, can be identified and mapped along the $\bar{\Gamma}-\bar{X}^{\prime}-\bar{M}-\bar{X}-\bar{\Gamma}$ boundary of the surface Brillouin zone (BZ). The resonance highest in energy is interpreted as due to the broken bond on the anion. Only weak emission from the surface resonances is observed in the first surface BZ. By comparing the experimental results with the calculation by Wang and Duke for $\mathrm{ZnS}(110)$, a description of the relaxation model for $\mathrm{CdTe}(110)$ is discussed.
\end{abstract}

\section{INTRODUCTION}

CdTe is the II-VI compound semiconductor which has attracted most interest in recent years. Much work has been devoted to the design of novel materials based on $\mathrm{CdTe}$, through, e.g., alloying with $\mathrm{Hg}$ during molecularbeam-epitaxial growth. In the course of this work, the need for accurate investigations of the electronic structure of CdTe has become a matter of great importance. Several investigations have been made in the past, both theoretical $^{1,2}$ and experimental. ${ }^{3-6}$ These previous descriptions need, in our opinion, more detail concerning the influence of the Cd $4 d$ states, the final states in the direct transitions and, especially important, the electronic structure of the (110) surface. The latter has direct consequences for the relaxation of the surface. A model for the surface geometrical structure has been proposed from low-energy electron diffraction (LEED) studies. ${ }^{7}$

Presented in this paper is an investigation of the valence-band structure of $\mathrm{CdTe}$ in the $\Gamma-(K)-X$ direction from which conclusions about the primary branches of the final band structure can also be drawn. An extensive study of the surface electronic structure of the (110) surface is also presented. In this, the advantages of using synchrotron radiation in angle-resolved uv photoelectron spectroscopy have been exploited. The continuous photon energy range and the high degree of linear polarization are the important qualities here.

\section{EXPERIMENTAL DETAILS}

A single-crystal bar, $5 \times 5 \times 20 \mathrm{~mm}^{3}$, of low resistivity $(\rho \sim 1000 \Omega \mathrm{cm})$ CdTe was held in a sample holder with five degrees of freedom and cleaved in situ at a pressure of $5 \times 10^{-10}$ torr. The crystal was oriented such that cleaving produced $5 \times 5 \mathrm{~mm}^{2}$ mirrorlike unreconstructed (110) surfaces of excellent order, as checked with LEED. Electron energy-distribution curves (EDC's) were recorded using an electron-energy analyzer, mounted on a twoaxis goniometer, and a multichannel analyzer with each channel normalized to the photon flux. Photons were supplied from the storage ring Doppel-Ring Speicheranlage (DORIS II) at Hamburger Synchrotronstrahlungslabor (HASYLAB), DESY in Hamburg, the Federal Republic of Germany, via the Seya-Namioka beamline. ${ }^{8}$ The total resolution, determined by the monochromator slit widths and the pass energy of the analyzer, was always better than $0.25 \mathrm{eV}$. The crystal was electrically connected to the sample holder through a GaAl alloy and the Fermi energy of the system, used as energy reference, was found by recording the Fermi edge of $\mathrm{Au}$, evaporated in situ on the sample holder.

Figure 1 shows the geometrical structure of the ideal, terminated $\mathrm{CdTe}(110)$ surface together with the surface and bulk Brillouin zone (BZ), the latter projected onto the (110) plane. Note that the plane normal to the surface and along the [001] direction is a mirror plane of the crystal.

\section{BAND-STRUCTURE CALCULATION}

We have used a combination of the linear augmentedplane-wave (LAPW) method $^{9}$ and the relativistic augmented-plane-wave (APW) method $^{10}$ to calculate the bulk band structure of CdTe. The LAPW scheme was used to perform a (nonrelativistic) self-consistent band- 

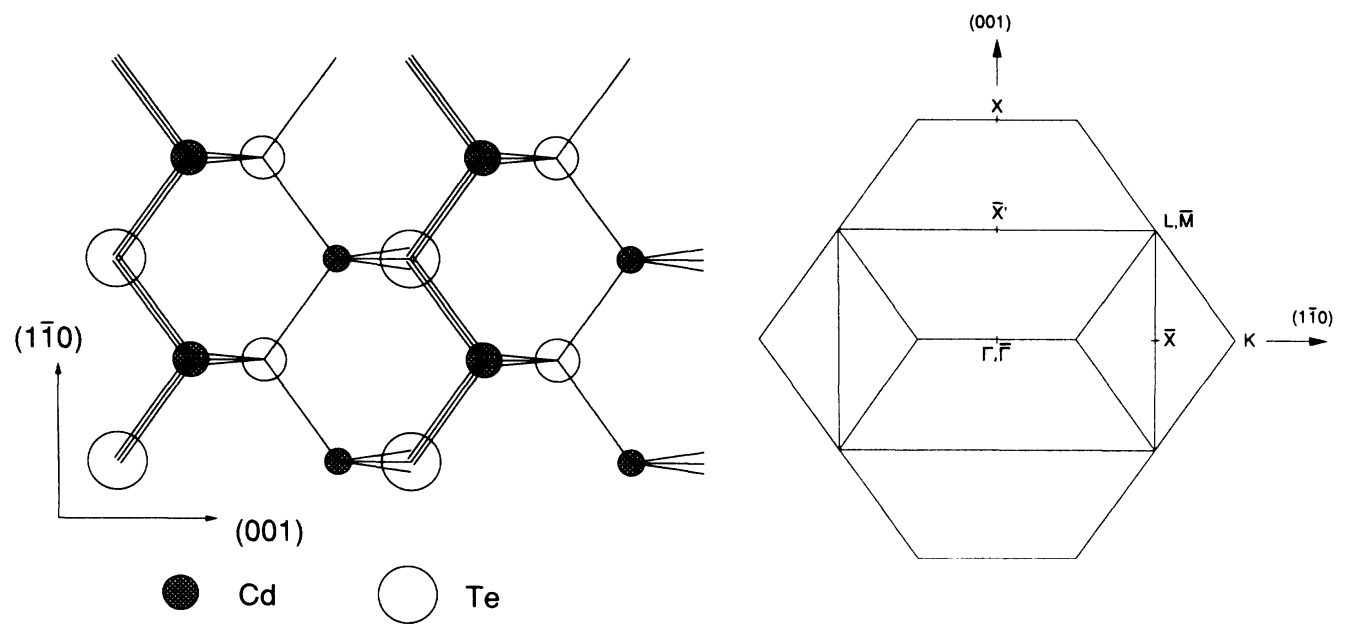

FIG. 1. The surface geometrical structure of the ideal, terminated CdTe(110) surface and the projected bulk Brillouin zone together with the $(1 \times 1)$ surface Brillouin zone.

structure calculation, in which we have used 89 unsymmetrized plane waves. The expansion in a spherical harmonics was taken up to $L=8$ and the energy parameters, $E_{1}$, used for the radial wave function inside the muffin tin, were all taken to be $0.2 \mathrm{Ry}$ below the valence-band maximum (VBM). This was found to give a convergence within a few $\mathrm{mRy}$ for the valence bands. The calculation was done within the local-density functional scheme and we have used the Hedin-Lundqvist approximation ${ }^{11}$ for the exchange and correlation potential. The nonspherical part of the potential inside the muffin tin was included by extending the plane waves into the muffin tin. This selfconsistent potential was then used to calculate the relativistic band structure, in this calculation 102 basis functions were used. The major difference between the two calculations, aside from the spin-orbit split of $0.95 \mathrm{eV}$ at the VBM, was a dramatic decrease of the fundamental band gap.

\section{RESULTS}

To investigate the bulk electronic structure along $\Gamma-(K)-X$ in the BZ, EDC's were recorded in normal emission $\left(\theta=0^{\circ}\right)$, with incidence angle of the light $\alpha=15^{\circ}$ and for photon energies, $h v$, from 13 to $26 \mathrm{eV}$. The polarization plane was held parallel with the mirror plane of the crystal. Figure 2 presents the recorded spectra. The peak positions of four structures have been marked in order to follow them through the series. In addition, a stationary structure is found at $\sim 6.2 \mathrm{eV}$ below the Fermi energy, $E_{F}$. An interesting emission is also found $\sim 1.5$ $\mathrm{eV}$ below $E_{F}$ in the $h v=13 \mathrm{eV}$ spectrum.

The peak positions, i.e., the initial-state energies of the assumed direct transitions, have in Fig. 3 been plotted versus photon energy together with theoretical structure curves as suggested by Williams et al. ${ }^{12}$ In the present analysis these curves are the (photon-)energy difference between the calculated initial, valence bands and the final bands, the latter being approximated by a free-electron parabolic band in the normal direction, with a band bottom of $E_{0}=-5.0 \mathrm{eV}$ relative to the valence-band max- imum. A value of $1.34 \mathrm{eV}$ was used for the difference between the Fermi energy and the VBM, in accordance with previous measurements. ${ }^{13}$ Using this scheme good agreement is found between the experimental data and the calculated valence bands. The structure in the spectra with photon energy $h v \leq 22 \mathrm{eV}$, dispersing downwards with increasing photon energy in the initial energy range around $-4 \mathrm{eV}$, is, however, not well described using the suggested final band. A similar analysis yields better agreement when a parabolic final band is folded in from the [111] direction and given a band bottom of $-8.0 \mathrm{eV}$. This structure is then identified as the spinorbit split second valence band counted from the gap down, with transitions observed from a region around the minimum in the zone.

Figure 4 shows the experimental data together with the calculated valence bands. The upper panel shows the free-electron final bands used, labeled with the appropriate reciprocal lattice vectors $\mathbf{G}$ according to the relation

$$
E(\mathbf{k})=\left(\hbar^{2} / 2 m_{e}\right)(\mathbf{k}+\mathbf{G})^{2}+E_{0} .
$$

The $\Gamma_{7}$ critical point is found $0.90 \mathrm{eV}$ below the $\Gamma_{8}$ point (VBM), as a result of spin-orbit interaction. The minimum of the spin-orbit split second valence band is found at $-3.0 \pm 0.25 \mathrm{eV}$ relative to the VBM, the large uncertainty being due to the crossing with a transition involving the second observed final-state band, in this region.

The stationary structure at $-6.2 \mathrm{eV}$ below $E_{F}$ in Fig. 2 shows a resonant behavior around $h v=21 \mathrm{eV}$. Considering the band structure as presented in Fig. 4, this structure is interpreted as due to emission from a high density of initial states on the flat part of the third valence band in the region between $K$ and $X$. The increased intensity is due to the transition passing through the $[G=(1,1,1)]$ like final-state band in the region around the $K$ and $X$ points.

In the investigation of the surface electronic structure, EDC's were recorded at selected photon energies and under specific polarization geometries, varying the emission 
angle along the high-symmetry azimuths which correspond to the $\bar{\Gamma}-\bar{X}^{\prime}$ and $\bar{\Gamma}-\bar{X}$ directions, as well as along the $\bar{X}^{\prime}-\bar{M}$ and $\bar{M}-\bar{X}$ directions of the surface BZ. A strong and necessary condition for assigning an observed structure to be of surface origin is the determination of its two dimensionality in the wave-vector dependence on the initial-state energy. A surface related structure must show no charge in its $E_{i}\left(k_{\|}\right)$dispersion when the photon energy and thus the $k_{\perp}$ value is changed. The dispersion should also show the periodicity of the surface BZ, which in the case of the unreconstructed (110) surface of zincblende structures is half that of the bulk BZ. These two criteria have been used in this study.

In Figs. 5(a) and 5(b) are presented two series of spectra, recorded along the $\bar{\Gamma}-\bar{X}^{\prime}$ direction, i.e., in the mirror plane. ${ }^{14}$ All marked features observed in the first surface

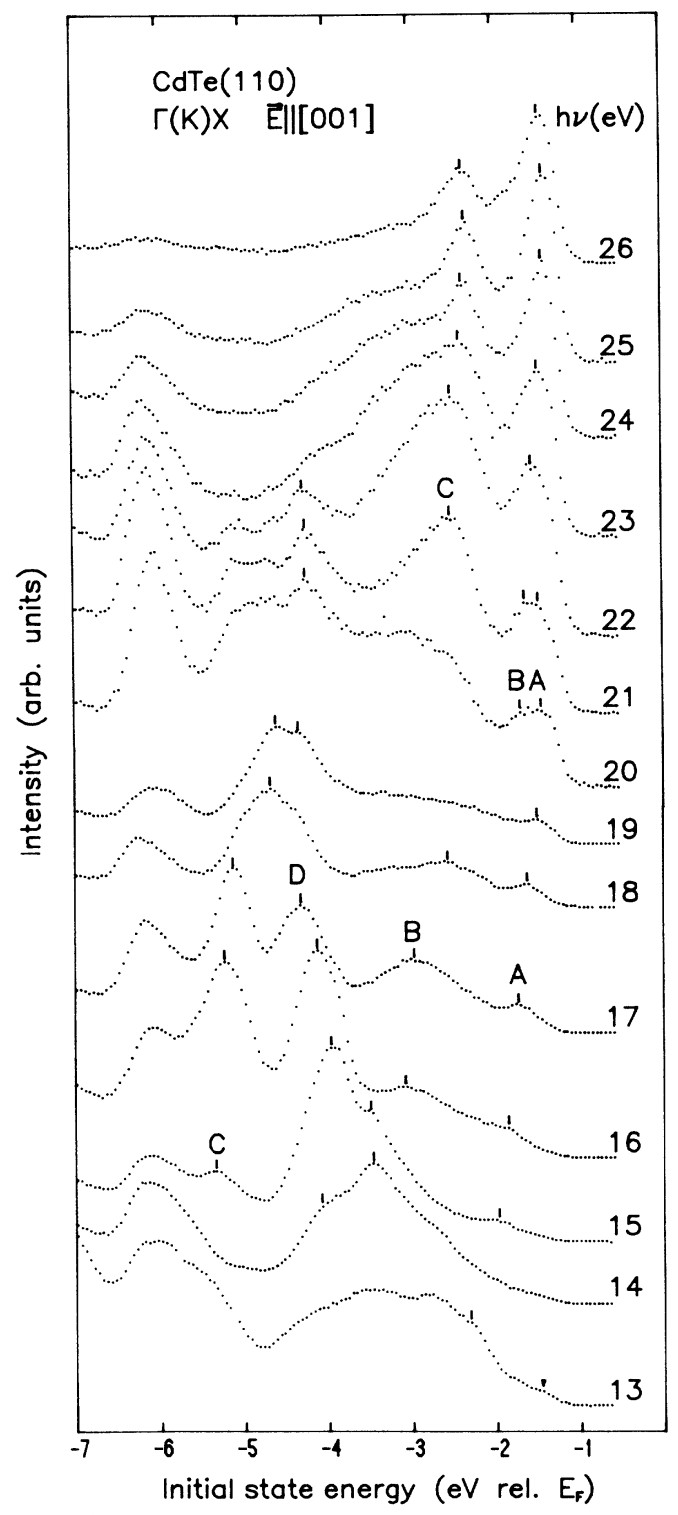

FIG. 2. Electron energy-distribution curves recorded at normal emission for various photon energies from the (110) surface of CdTe. The angle of incidence is $\alpha=15^{\circ}$ and the polarization is held perpendicular to the [001] direction.

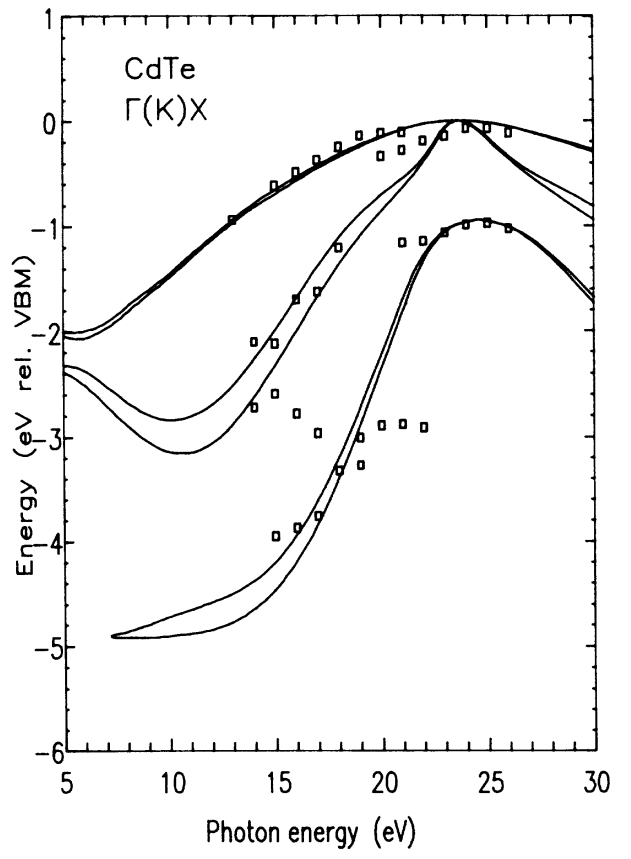

FIG. 3. Structure plot for the results of Fig. 2, open squares, together with the theoretical curves of assumed direct transitions from the calculated APW valence bands to free-electron final bands in the normal direction.

BZ $\left(\bar{\Gamma}-\bar{X}^{\prime}\right)$ are clearly of bulk origin, since they show initial-state energy dispersion with respect to photon energy. Furthermore, as the two upper structures reach $\bar{X}^{\prime}$, they continue dispersing down in energy, showing no periodicity with the surface BZ. However, at all investigated photon energies, two new structures, labeled $S_{1}$ and $S_{2}$, appear at $\bar{X}^{\prime}$ dispersing upwards, go through a maximum at $\bar{\Gamma}_{2}$ and continue dispersing down in energy. These structures show the periodicity of the surface $\mathrm{BZ}$ and, what is more important, have the same $E_{i}\left(k_{\|}\right)$ dispersion independent of photon energy, within the experimental inaccuracy. The upper structure, $S_{1}$, is most pronounced, but the interpretation that both are twodimensional, surface related features is clear. Their energy-positions at $\bar{\Gamma}$ are $-0.3 \mathrm{eV}$ and $-0.9 \mathrm{eV}$ relative to the VBM, respectively, which determines them as being surface resonances. This result explains the emission found just below the VBM at $h v=13 \mathrm{eV}$ in Fig. 2 as being due to the upper surface resonance.

The structure $S_{4}$, most clearly seen in the $h v=18 \mathrm{eV}$ series [Fig. 5(b)], is here only appearing at emission angles where the region around $\bar{X}^{\prime}$ is probed. The dispersion of this structure can be further followed in the series of spectra recorded along $\bar{X}^{\prime}-\bar{M}$, shown in Fig. 5(c). As we here approach $\bar{M}$ a very sharp structure labeled $S_{3}$ is observed dispersing down, symmetrically around $\bar{M}$. From the bulk band calculation we find the $S_{3}$ and $S_{4}$ structures to be situated in the empty lens of the projected bulk band structure, which exists along $\bar{X}^{\prime}-\bar{M}-\bar{X}$ in the surface BZ. These structures are accordingly interpreted as true surface states on the CdTe(110) surface, i.e., states localized to the surface which at least partially 
exist in energy regions where bulk states are absent.

Investigation of the surface electronic structure along $\bar{\Gamma}-\bar{X}$ was done analogously to what has been described above. Since this is not in a mirror plane of the crystal structure, no attempt was made to separate out bulk contributions using polarization selection rules. An angular series at $22-\mathrm{eV}$ photon energy is presented in Fig. 5(d). Also here we find all marked features observed from $\bar{\Gamma}-\bar{X}$, i.e., in the first surface $B Z$, to be of bulk origin. They show dispersion with respect to photon energy and have the periodicity of the bulk lattice. At $\bar{X}$ we note the appearance of a weaker structure, labeled $S_{1}$, dispersing up towards $\bar{\Gamma}_{2}$. This structure is also observed at photon energies 16 and $18 \mathrm{eV}$ and is accordingly suggested as being due to emission from a surface related state. Thus we find this to be the continuation of the upper surface resonance found along $\bar{\Gamma}-\bar{X}^{\prime}$. A weak shoulder $S_{2}$ can be observed where the second surface resonance is expected to appear.

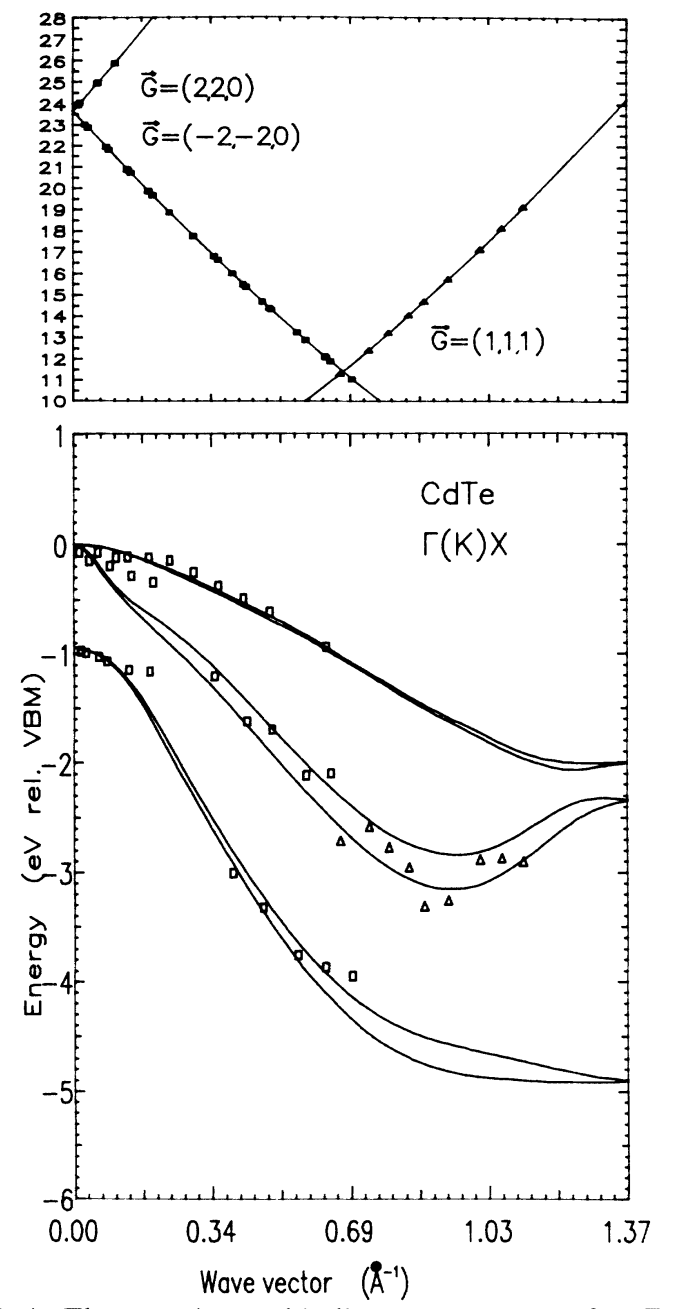

FIG. 4. The experimental bulk band structure of CdTe along $\Gamma-(K)-X$ shown together with the calculated APW valence bands. The upper panel shows the final-state bands, labeled with their reciprocal lattice vectors. Transitions involving the $(-2,-2,0)$ and $(2,2,0)$ bands are indicated with squares while triangles indicate transitions to the $(1,1,1)$ band.
Characterization of the observed surface states and resonances in terms of their dependence on polarization and on the inequivalent emission directions within the mirror plane, is presented in Fig. 6. Spectra $a$ and $b$ show the $p_{z}$ dependence of $S_{1}$ and $S_{2}$ ( $z$ is in the surface normal direction). Both structures show strong intensity enhancement when the electrical field of the incident light becomes more parallel with the surface normal, i.e., with increasing $p_{z}$ character. Spectra $c$ and $d$ probe the first $\bar{X}^{\prime}$ point of the surface BZ but on opposite sides of $\bar{\Gamma}$. They show the emission from $S_{4}$ to be strongest in the direction which is opposite the Te dangling bond.

Figure 7 summarizes the experimental surface electronic structure of $\mathrm{CdTe}(110)$ by showing the observed dispersions along the $\bar{\Gamma}-\bar{X}^{\prime}-\bar{M}-\bar{X}-\bar{\Gamma}$ boundary of the surface $B Z$ together with the projection of the relativistic APW valence-band structure. Energies are referred to the VBM. The energy value for $S_{1}$ at $\bar{\Gamma}$ is $-0.3 \mathrm{eV}$, at $\bar{X}^{\prime},-0.85 \mathrm{eV}$, and at $\bar{X},-0.9 \pm 0.1 \mathrm{eV}$, by extrapolation. $S_{2}$ is found at $-0.9 \mathrm{eV}$ at $\bar{\Gamma} . S_{3}$ has its minimum energy value at $\bar{M},-3.2 \mathrm{eV}$, while $S_{4}$ is found at $-3.0 \mathrm{eV}$ at the $\bar{X}^{\prime}$ point and at $-4.6 \mathrm{eV}$ at the $\bar{M}$ point.

\section{DISCUSSION}

In the interpretation of the normal emission data we have chosen to use free-electron parabolic final states in order to determine the $k_{\perp}$ values of the observed transitions. This can be motivated in several ways, but we merely state that this can, at most, be a good approximation of a band passing through the true final-state bands, to which strong coupling by the photon field to the initial state occurs in the experiment under consideration. Figures 3 and 4 give evidence that the procedure is a good approximation in the case of CdTe in the $\Gamma-(K)-X$ direction. The calculated valence bands account well for the experimentally found structures and correctly predict the spin-orbit splitting of the $\left(\Gamma_{7}, \Gamma_{8}\right)$ points. Our experimental normal emission results agree well with those of Silberman et al. ${ }^{5}$ The apparently better resolution of the present work allows a more accurate mapping of the bands near the VBM, as well as a deepened analysis of the final bands involved.

The investigation of the surface electronic structure of the cleaved (110) surface of CdTe presented in this paper is not the first investigation ever conducted. Ebina et al. ${ }^{3}$ have conducted a study of $\mathrm{CdTe}(110)$ using $\mathrm{He} \mathrm{I}, h v$ $=21.2 \mathrm{eV}$, for the excitation. The upper surface resonance, $S_{1}$, mapped along $\bar{\Gamma}-\bar{X}^{\prime}$ and $\bar{\Gamma}-\bar{X}$ in the present study, is also observed in the study by Ebina et al. ${ }^{3}$ They suggest it to be due to a surface state on the grounds that it shows periodicity with the surface BZ in its initial-state energy dispersion. In the study presented in this paper, the possibility at a synchrotron radiation source to choose the photon energy more freely has been used to determine the lack of dispersion of this structure with $k_{\perp}$, which in our opinion is the fundamental condition.

Our normal emission data shows the energy position of the upper valence band at $21-\mathrm{eV}$ photon energy to be 0.15 $\mathrm{eV}$ below the VBM. This is close to the energy position of the upper surface resonance $S_{1}$, by coincidence, and 
this has lead Pessa et al. ${ }^{4}$ to the assumption that the structure found closest to the VBM in normal emission at 21.2-eV photon energy is the structure which Ebina et $a l .{ }^{3}$ suggest to be of surface origin. Pessa et al. ${ }^{4}$ claim to observe surface sensitivity in this structure, but from the present results there is no doubt that changes in emission intensity of this peak reflects primarily the bulk structural order.

The $S_{2}$ surface resonance was not observed by Ebina et $a l .{ }^{3}$ In this paper the surface origin of this structure is determined using the same criteria as for $S_{1}$. Both $S_{1}$ and $S_{2}$ show rather strong $p_{z}$ character. Further, we find the same $\left(p_{x}, p_{y}\right)$ character for $S_{1}$ as for the top valence band. In a previous study of CdS (Ref. 15) a surface resonance strongly related to the top valence band was associ- ated with the strongly ionic, broken bond on the anion. We interpret $S_{1}$ in a similar manner, with the qualitative distinction that the results indicate more $p_{z}$ character of the broken bonds on $\mathrm{CdTe}(110)$. The association of a specific bond for $S_{2}$ cannot be made from this study.

A description of the previously unobserved surface states $S_{3}$ and $S_{4}$ in terms of orbital character was not possible based on the experiment alone. We note, however, that $S_{4}$ is quite similar to the $B_{2}$ surface state found on GaAs(110) by Huijser et al. ${ }^{16}$ This $B_{2}$ surface state is described by Pandey et al. ${ }^{17}$ as being of mixed anion $p_{x, y}$ and cation $s$ character.

In a recent paper by Wang and Duke, ${ }^{18}$ the surface electronic structure of $\mathrm{ZnS}(110)$ has been calculated within a tight-binding scheme. The results of the calcula-
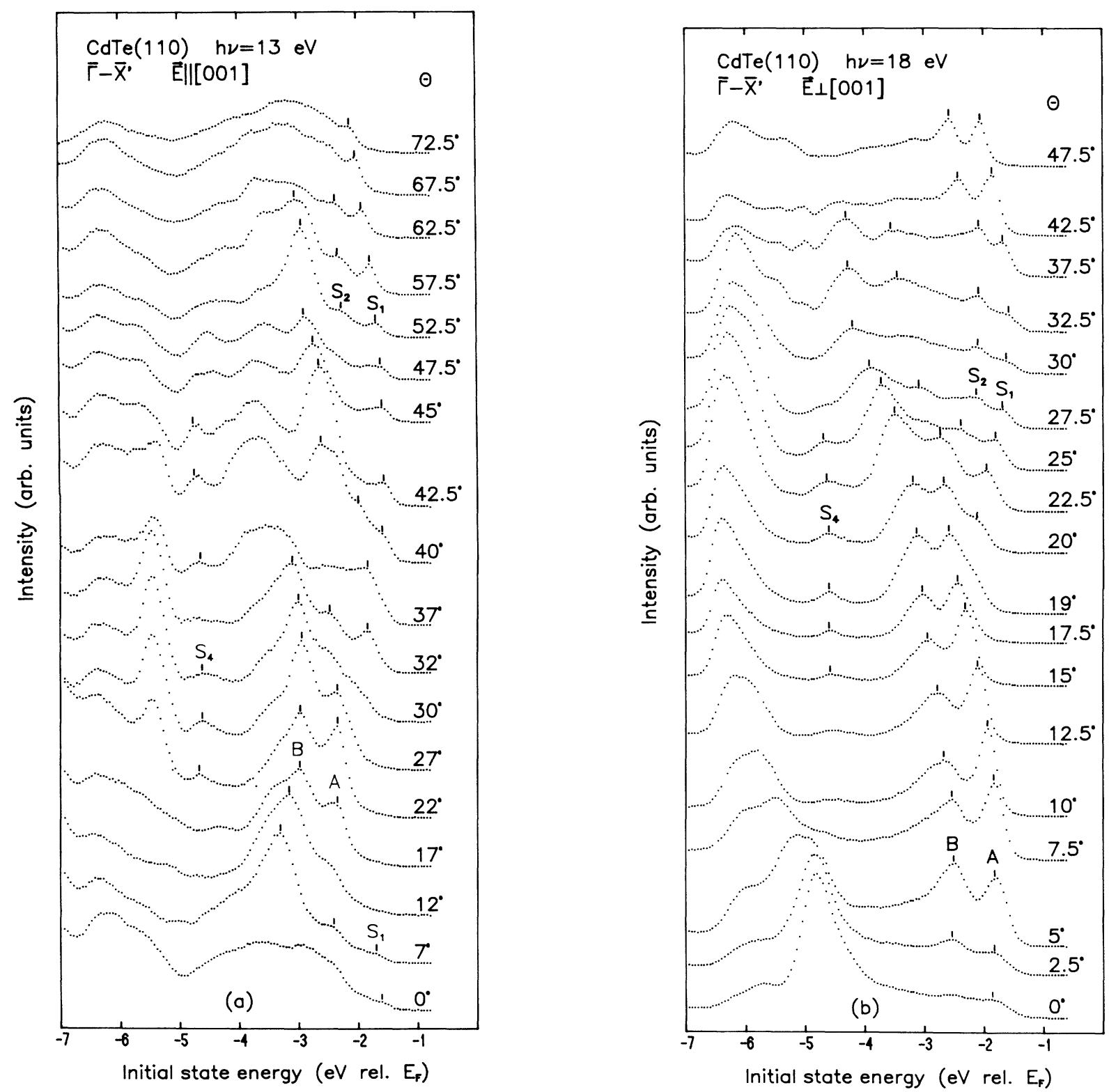

FIG. 5. Electron distribution curves recorded at various angles of emission $\theta$, probing states along high-symmetry directions in the surface BZ. (a) the $\bar{\Gamma}-\bar{X}^{\prime}[001]$ azimuth at $13-\mathrm{eV}$ photon energy, $\alpha=15^{\circ}$, (b) the $\bar{\Gamma}-\bar{X}^{\prime}[001]$ azimuth at $18-\mathrm{eV}$ photon energy, $\alpha=44^{\circ}$, (c) the $\bar{X}^{\prime}-\bar{M}$ direction at $16-\mathrm{eV}$ photon energy, $\alpha=45^{\circ}$, and (d) the $\bar{\Gamma}-\bar{X}[1 \overline{1} 0]$ azimuth at $22-\mathrm{eV}$ photon energy, $\alpha=45^{\circ}$. 

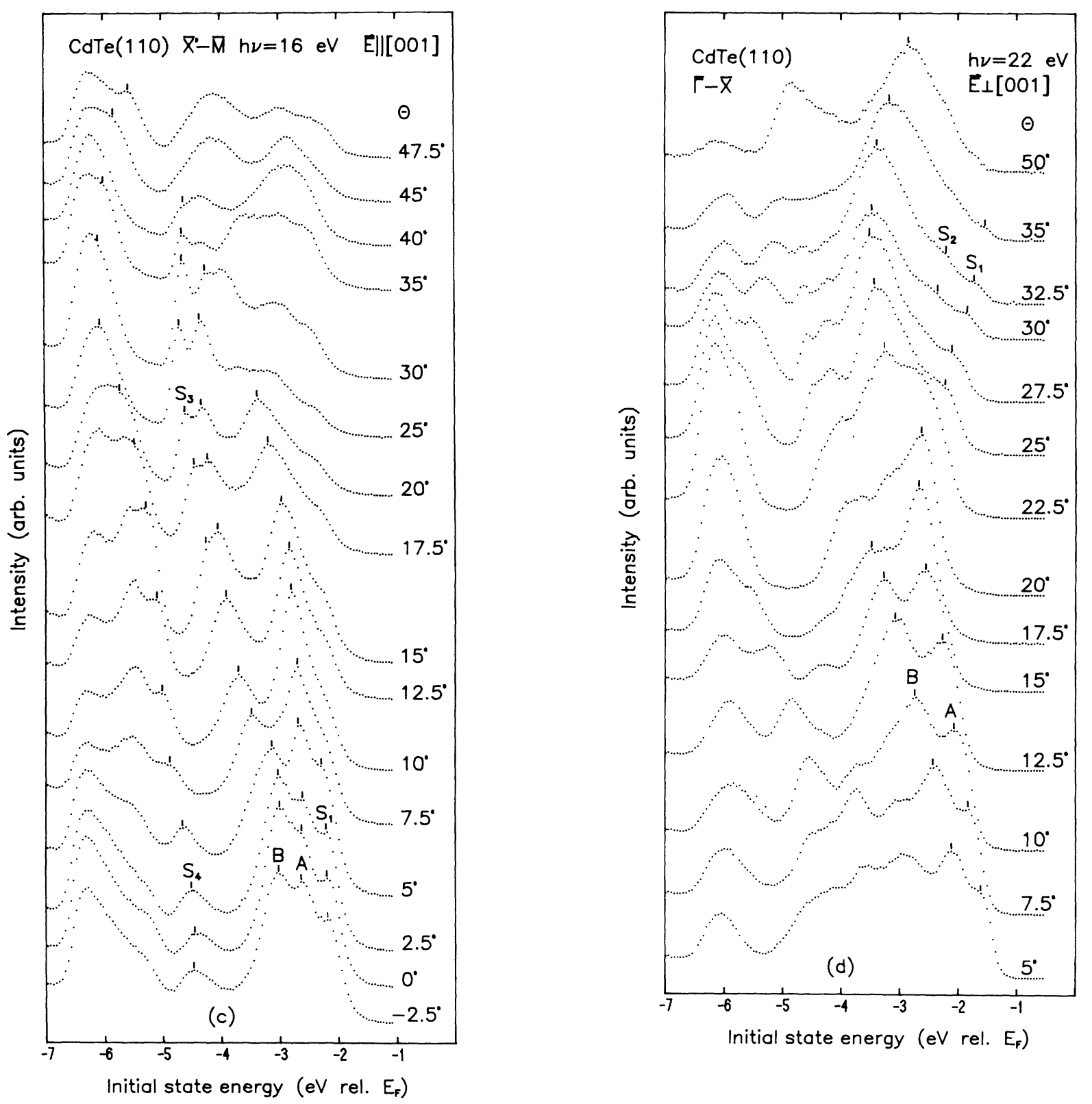

FIG. 5. (Continued).

tion indicate the presence of four surface states or resonances on this surface. These have dispersions along the boundary of the surface BZ which are qualitatively similar to the experimental results of this work on $\mathrm{CdTe}(110)$. In the calculation, the structure $S_{1}$ is a surface state everywhere except close to $\bar{\Gamma}$, while we find a similar state as a surface resonance, at least along $\bar{X}-\bar{\Gamma}-\bar{X}^{\prime}$. The experiment further shows a somewhat different dispersion for the $S_{3}$ surface state. The paper by Wang and Duke ${ }^{18}$ also presents the orbital character of the four structures.

\section{SUMMARY}

Using angle-resolved uv photoelectron spectroscopy, an investigation of the bulk and surface electronic struc- ture of $\mathrm{CdTe}(110)$ has been performed. A calculation of the valence-band structure of $\mathrm{CdTe}$ using a relativistic APW formulation, where the shallow $\mathrm{Cd} 4 d$ states have been included, is presented for comparison with the results of the experiment.

Modeling the final bands of the observed direct transitions from the valence bands along $\Gamma-(K)-X$ with freeelectron parabolic bands in the normal direction and folded in from the [111] direction, yields a good agreement of the calculated bands with the experimental data. The $\Gamma_{7}$ critical point is found $0.9 \mathrm{eV}$ below the $\Gamma_{8}$ maximum of the valence band, as a result of spin-orbit interaction.

The surface electronic structure has been mapped along the $\bar{\Gamma}-\bar{X}^{\prime}-\bar{M}-\bar{X}-\bar{\Gamma}$ boundary of the surface $\mathrm{BZ}$, 


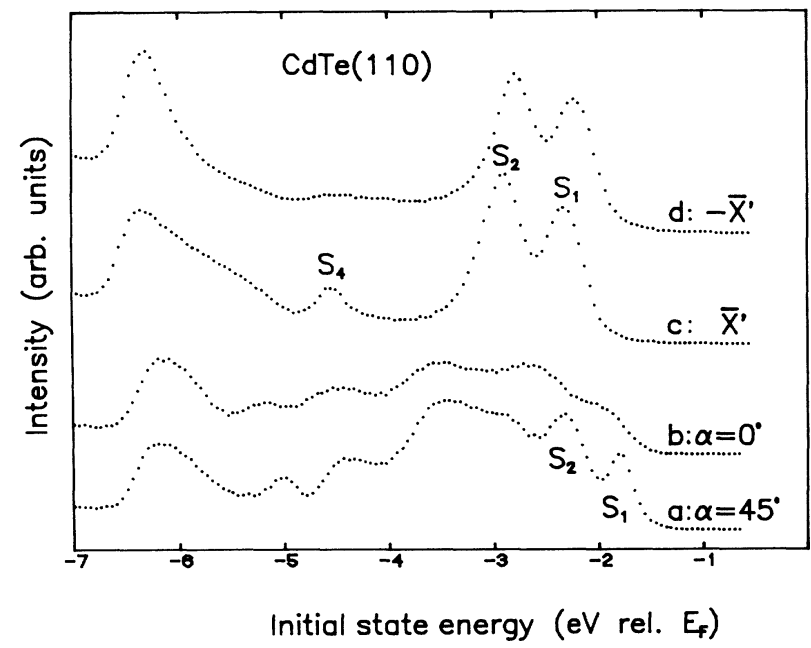

FIG. 6. Electron distribution curves recorded to investigate the dependence on polarization and emission direction of the found surface states and resonances ( $\mathbf{E}$ is parallel to [001]): $a$, $h v=16 \mathrm{eV}, \theta=50^{\circ}, \alpha=45^{\circ} ; b, h v=16 \mathrm{eV}, \theta=50^{\circ}, \alpha=0^{\circ} ; c$, $h v=16 \mathrm{eV}, \alpha=45^{\circ}, \theta=-17.5^{\circ}\left(-\bar{X}^{\prime}\right) ; d, h v=16 \mathrm{eV}, \alpha=45^{\circ}$, $\theta=17.5^{\circ}\left(\bar{X}^{\prime}\right)$. (The shift of the initial-state energy values for $S_{1}$ and $S_{2}$ is only due to the inexact crystal orientation, resulting in the probing of slightly different $k_{\|}$points.)

through the use of two criteria which must be fulfilled by surface states and resonances: no initial-state energy dispersion with respect to $k_{1}$; periodicity in the dispersion according to the surface BZ. Two surface resonances, $S_{1}$ and $S_{2}$, have been observed. Both have downward dispersion away from $\bar{\Gamma}$ and are found within the projected bulk bands. Their energy positions at $\bar{\Gamma}$ are -0.30 and $-0.90 \mathrm{eV}$ (relative to the VBM), respectively. Through the observed polarization dependence we interpret $S_{1}$ as due to the broken bond on the anion.

Two true surface states, i.e., surface states in a gap of the projected bulk band structure, are found in the empty lens extending from $\bar{X}^{\prime}$ to $\bar{M}$ and $\bar{X}$ in the surface $\mathrm{BZ}$.

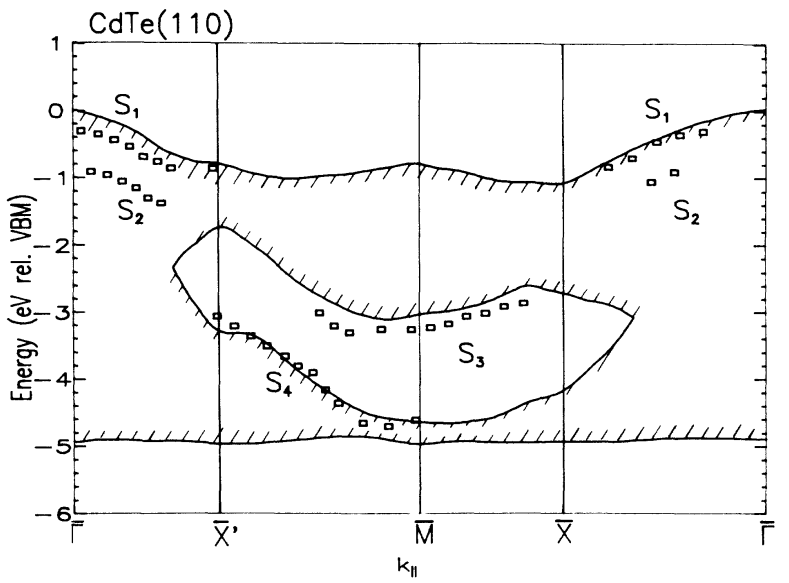

FIG. 7. The experimental surface electronic band structure along the $\bar{\Gamma}-\bar{X}^{\prime}-\bar{M}-\bar{X}-\bar{\Gamma}$ boundary of the surface $\mathrm{BZ}$ of $\mathrm{CdTe}(110)$. The cross-hatched areas indicate the projected APW valence bands.

The surface state labeled $S_{4}$ shows strong emission intensity in the direction opposite the ideal Te dangling bond direction. The description of the $S_{3}$ surface state is not clear.

Wang and Duke ${ }^{18}$ present a relaxation model for the (110) surface of $\mathrm{ZnS}$ in which the anion is displaced out of the surface plane and the cation into the same. Within a tight-binding formulation they calculate the dispersion of four surface states which show rather strong similarities with the results presented in this paper on CdTe. We would encourage a calculation of the surface electronic band structure within a similar relaxation model for the (110) surface of CdTe.

\section{ACKNOWLEDGMENTS}

We thank Dr. M. Lähdeniemi and the staff at HASYLAB for their excellent technical assistance. The experimental cooperation of Dr. P. Mårtensson and S. Wiklund is gratefully acknowledged. This work was supported by the Swedish Natural Science Research Council.
*Present address: Physics Laboratory III (Materials Science), Royal Institute of Technology, S-10044 Stockholm 70, Sweden.

1J. R. Chelikowsky and M. L. Cohen, Phys. Rev. B 14, 556 (1976).

${ }^{2}$ C. Calandra and G. Santoro, J. Vac. Sci. Technol. 13, 773 (1976).

${ }^{3} A^{*}$. Ebina and T. Takahashi, J. Cryst. Growth 59, 51 (1982).

${ }^{4}$ M. Pessa, P. Huttunen, and M. A. Herman, J. Appl. Phys. 54, 6047 (1983).

${ }^{5}$ J. A. Silberman, D. Laser, C. K. Shih, D. J. Friedman, I. Lindau, W. E. Spicer, and J. A. Wilson, J. Vac. Sci. Technol. A 3, 233 (1984).

${ }^{6}$ T. P. Humphreys, G. P. Srivastava, and R. H. Williams, J. Phys. C 19, 1259 (1986).

${ }^{7}$ C. B. Duke, A. Paton, W. K. Ford, A. Kahn, and G. Scott, Phys. Rev. B 24, 3310 (1981).
${ }^{8}$ C. A. Feldman, R. Engelhardt, T. Permien, E. E. Koch, and V. Saile, Nucl. Instrum. Methods 208, 785 (1983).

${ }^{9}$ O. K. Anderson, Phys. Rev. B 12, 3060 (1975).

${ }^{10}$ See, e.g., T. L. Loucks, Augmented Plane Wave Method (Benjamin, New York, 1967).

${ }^{11}$ L. Hedin and B. I. Lundqvist, J. Phys. C 4, 2064 (1971); see also U. von Barth and L. Hedin, ibid. 5, 1629 (1972).

${ }^{12}$ G. P. Williams, F. Cerrina, G. J. Lapeyre, J. R. Anderson, R. J. Smith, and J. Hermanson, Phys. Rev. B 34, 5548 (1986).

${ }^{13}$ K. O. Magnusson, U. O. Karlsson, D. Straub, S. A. Flodström, and F. J. Himpsel, Phys. Rev. B 36, 6566 (1987).

${ }^{14}$ The Fermi energy was not recorded at $13 \mathrm{eV}$ photon energy. The energy scale in Fig. 5(a) has been chosen by lining up the maximum initial-state energy of the structure $S_{4}$ with the value observed at the three other investigated photon energies, these being properly referenced with respect to the Fermi energy. 
${ }^{15}$ K. O. Magnusson, S. A. Flodström, P. Mårtensson, J. M. Nicholls, U. O. Karlsson, R. Engelhardt, and E. E. Koch, Solid State Commun. 55, 643 (1985).

${ }^{16}$ A. Huijser, J. van Laar, and T. L. van Rooy, Phys. Lett. 65A,
337 (1978).

${ }^{17}$ K. C. Pandey, J. L. Freeouf, and D. E. Eastman, J. Vac. Sci. Technol. 14, 904 (1977).

${ }^{18}$ Y. R. Wang and C. B. Duke, Phys. Rev. B 36, 2763 (1987). 